\title{
In Search of an Ideal Obesity Assessment Tool : Is Body Mass Index Reliable Enough?
}

\author{
Vidyashree S. Hulkoti' ${ }^{1}$, Sourya Acharya ${ }^{2}$, Samarth Shukla ${ }^{3}$, Sree Karthik Partapa ${ }^{4}$, Yash Gupte ${ }^{5}$ \\ ${ }^{1}$ Department of Medicine, DMIMS (Deemed to Be University), Sawangi (Meghe), Wardha, \\ Maharashtra, India. ${ }^{2}$ Department of Medicine, DMIMS (Deemed to Be University), Sawangi \\ (Meghe), Wardha, Maharashtra, India. ${ }^{3}$ Department of Pathology, DMIMS (Deemed to Be \\ University), Sawangi (Meghe), Wardha, Maharashtra, India. ${ }^{4}$ Department of Medicine, DMIMS \\ (Deemed to Be University), Sawangi (Meghe), Wardha, Maharashtra, India. ${ }^{5}$ Department of \\ Medicine, DMIMS (Deemed to Be University), Sawangi (Meghe), Wardha, Maharashtra, India.
}

\section{ABSTRACT}

\section{BACKGROUND}

Obesity, a global pandemic, has become a chronic health problem within a modern western society. Obesity mimics the iceberg phenomenon wherein there is more to it than what we perceive resulting in various physical and psychological problems. Obesity is defined as surplus body weight for given height. . Obesity has been triggered by the growth of economy caused as an effect of industrialization, and urbanization, associated sedentary lifestyle, and transition of nutrition to canned foods. In the last few years, globally, countries have witnessed the spike in the rate of obesity. The endangering effects presented by obesity lead to numerous comorbidities that are being masked by the body dysmorphism. Metabolic disorders like diabetes mellitus type 2 and various cardiovascular risks hamper the regular metabolism of the body. Exploring the cascading effects in changing sedentary lifestyles draws many parallels to the surge in overweight and obesity among the people following such lifestyles. Increased adoption of sedentary lifestyles has resulted in a cascading effect on various metabolic disorders associated with obesity, globally. To address this surging concern, researchers around the globe have come up with multiple indices and parameters such as BMI, ABSI, VAI, BIA, DEXA, waist-hip ratio, and waist circumference, to quantify obesity in one final equation. However, these parameters have failed to give a conclusive summation that helps to identify the pre-symptoms of obesity. Similarly, variations in physical size and different body compositions for different weight categories usually pose tremendous challenges to quantify obesity. To make things more complicated various forms of obesity are being described and each has got its implication as far as the development of cardiovascular burden is concerned.

This challenge presents the need to derive and identify a much robust, accurate and explicit index that would apply universally to all forms of obesity and would guide preventive and therapeutic strategies thereoff. In this article, an effort is being made to compare various parameters available globally to tail off the better and more reliable indicator available.

\section{KEY WORDS}

Obesity, Pandemic, Cardiovascular, Diabetes Mellitus

\author{
Corresponding Author: \\ Dr. Sourya Acharya, \\ Professor, \\ Department of Medicine, \\ DMIMS (Deemed to Be University), \\ Sawangi (Meghe), Wardha, \\ Maharashtra, India. \\ E-mail: souryaacharya74@gmail.com
}

DOI: $10.14260 /$ jemds/2020/555

How to Cite This Article:

Hulkoti VS, Acharya S, Shukla S, et al. In search of an ideal obesity assessment tool: is body mass index reliable enough? J Evolution Med Dent Sci 2020;9(35):25562560, DOI: $10.14260 /$ jemds/2020/555

Submission 22-04-2020,

Peer Review 14-07-2020,

Acceptance 21-07-2020,

Published 31-08-2020.

Copyright (C) 2020 JEMDS. This is an open access article distributed under Creative Commons Attribution License [Attribution 4.0 International (CC BY 4.0)] 


\section{BACKGROUND}

\section{Tools to Evaluate Obesity / Body Mass Index}

Overweight and obesity are global non-communicable pandemics. Obesity may be explained as percentage of fat storage linked with increased health risks and complications. Nonetheless, as the fat mass is laborious to quantify, the efficient definition of obesity is stationed on the Body Mass Index (BMI). Recent studies hint that mean BMI levels are expeditiously increasing presumably due to increasing motorization leading to scaled down physical activity and easy accessibility of processed foods. ${ }^{1}$ In the year 1835, a Flemish astronomer, Lambert Quetelet described BMI also known as Quetelet's index. ${ }^{2,3}$ The BMI is calculated as weight in kilograms divided by height in meter squared. It is widely adopted to quantify obesity. ${ }^{4}$

\section{Limitations of BMI}

BMI fails to demarcate between the fat and lean body mass. As a matter of fact, any individual with a high BMI may have a low fat mass and vice versa. The term obesity is related to the surplus accumulation of the body fat and on this basis, the effectiveness of BMI as a marker of body fat mass has been questioned time and again. In population-based studies, men usually have BMI higher than in women.5,6 BMI is an indirect tool of body fat correlated with bioelectrical impedance. In addition, BMI lacks to resonate the modifications that occur with age. Further, BMI as a gauge of obesity can preface the various problems that may lead to potent bias in evaluating the effects associated with obesity. ${ }^{7}$ The rather inefficient relationship between BMI and the percent of body fat mass has been stated clearly in the database of NHANES III. ${ }^{8}$ It has been observed that many obese people have cardiovascular risk factors, and their BMI do not correlate with cardiovascular events. ${ }^{9-11}$ In the observational study of EPIC, ${ }^{12}$ the waist circumference-to-BMI ratio was calculated and was observed that the ratio was highest in the individuals who had low BMI.

\section{Waist Circumference, Waist Hip Ratio and Waist-Height Ratio:}

The Waist Circumference (WC) complements BMI for the analysis of disease risk in obesity. Abdominal fatness is an individualistic prognostic marker indicative of comorbidities and mortality. In 201713 International Chair on Cardiometabolic Risk (ICCR) and the International Atherosclerosis Society (IAS) working group on visceral obesity highlighted the significance of abdominal obesity as a marker for premature atherosclerosis and cardiovascular diseases in adults. The group developed a consensus statement which outlined that; BMI alone is not sufficient to estimate the cardio metabolic risk related to increased adiposity. Measuring waist circumference should be considered as a routine habit in clinical practice along with the BMI. Studies have proved that, adults with higher waist circumference values are at higher risk than those having lower waist circumference irrespective of any given BMI category. ${ }^{14,15}$ Margaret Ashwell and others proposed the waist-height ratio as a measure of abdominal obesity. ${ }^{16,17}$ Compared with the previous measurements, the waist-height ratio shows similar and sometimes slightly stronger associations with the risk of Cardiovascular disease (CVD) or Type 2 diabetes mellitus (T2DM). ${ }^{18}$ An explanation for why adding height increases the prediction of disease risk might be because short stature is associated with increased risk of CVD. ${ }^{19}$ Waist circumference is an easy tool to measure abdominal adiposity, and correlates well with all-cause, and CVD mortality. ${ }^{20-23}$

\section{Consensus Statement ${ }^{24}$}

The consensus statement for obesity in India has described various cut-off (action) levels for defining and managing overweight and obesity. The gravity of waist circumference in estimating the various cardio metabolic risk factors and its outcomes has been inspected in various epidemiological studies. $^{25-27}$ The association of waist circumference and its clinical outcome is persistently amplified for diabetes risk, and waist circumference is a potent indicator of diabetes mellitus than is body mass index. It has been noted that waist circumference is a better indicator to identify individuals who are at risk than the body mass index alone. Data from different studies pillars the idea that waist circumference is a principal marker of diabetes, coronary heart disease and is independent of the native clinical tests like blood pressure, random blood sugar, and lipoproteins. ${ }^{28,29}$

Waist circumference serves as an optimistic marker of health outcomes. In fact, the relationship between waist circumference and health outcome changes very little with the increasing age in comparison with its association of BMI and health outcome. According to a research that compared the sensitivity of different variables towards cardiovascular disease, the study supports that abdominal obesity is more sophisticated risk indicator of CVD than BMI. Pratyush DD et $\mathrm{al}^{30}$ studied the relation of different individual criteria of metabolic syndrome (MetS) with the suitable waist circumference cut-off for South Asian Indians. MetS was diagnosed by modified NCEP ATP III criteria. $90 \mathrm{~cm}$ was the waist circumference cut-off point for male as per the receiver operator characteristic curve analysis and was associated with MetS in males with sensitivity and specificity of $71 \%$ and $96 \%$ respectively; and for females was $85 \mathrm{~cm}$ with a sensitivity and specificity of $86 \%$ and $93 \%$, respectively.

Snehalatha C; ${ }^{31}$ studied the risk of diabetes with BMI, WC, or waist hip ratio (WHR). They performed oral glucose tolerance tests in all subjects. It was observed that the cut-off values for WC and WHR were lower in women than in men. Ghosh JR32; study examined the relationship between the varied adiposity measures and figured out the optimum adiposity measure available in interpreting Type 2 DM. 187 adult males aged 25-67 years were included. Fasting and two hours post prandial glucose levels were assessed. Highest odds ratio for Type $2 \mathrm{DM}$ was observed for the waist circumference. This study concluded that, waist circumference was the ideal tool for measurement of the central adiposity and assessing Type 2 DM. ${ }^{32}$
A Body Shape Index
A latest anthropometric measure (A Body Shape Index, ABSI) in concurrence with BMI can calculate the visceral abdominal and also the general adiposities. ${ }^{[33]}$ It is defined as the waist 
circumference (WC) / (BMI (Body Mass Index) $2 / 3 \times$ height $1 /$ ${ }^{2}$ ). Linear and positive association of ABSI has also been indicated with visceral fat mass and the cardiovascular disease (CVD). Updated studies also confirms that ABSI is a strong indicator of all-cause mortality. ${ }^{34} \mathrm{~A}$ European study confirmed that ABSI and BMI could forecast the probability of CVD events more effectively than any of the other indices. ${ }^{35}$ The findings in the studies, ${ }^{36-37}$ suggests that it is of utmost priority that ABSI shows a linear relationship with all-cause or cardiovascular mortality, while BMI and WC show J-shaped associations. ${ }^{38,39}$

\section{Visceral Adiposity Index}

The visceral adiposity index (VAI) is an accurate tool used to calculate metabolic abnormality using waist circumference, serum triglycerides, age, and gender. The visceral adiposity index is also considered a proxy marker for cardio metabolic risk.

\section{Calculation}

VAI is calculated in males and females separately.

Males $=($ WC $39.68+1.88 \times$ BMI $) \times($ TGs 1.03 $) \times(1.31 \mathrm{HDL}-\mathrm{C})$

Females $=($ WC $36.58+1.89 \times$ BMI $) \times($ TGs 0.81$) \times(1.52$ HDL - C)

Where,

WC is expressed in $\mathrm{cm}, \mathrm{BMI}$ in $\mathrm{Kg} / \mathrm{m}^{2}$, TG in $\mathrm{mmol} / \mathrm{L}$, and HDL in $\mathrm{mmol} / \mathrm{L}$

Further analysis of multiple such data points derived that VAI should be equivalent to 1 for healthy non-obese subjects with normal adipose distribution and normal TG and HDL levels. VAI is also useful to determine fat distribution which can be further detrimental to the phenotype change and minimalizes the need to take expensive imaging tests, thus delivering a more idealistic approach to investigate various cardio metabolic risks associated with visceral obesity. ${ }^{40}$ Also, VAI shows a correlation with known adipocytokines and cardio metabolic risk serum markers.

An aspect that is worthy to be challenged is the changes in the VAI on a calorie deficient diet. However, in theory a healthy weight loss program with a minimal reduction in the caloriebalanced diet along with aerobic exercise decreases VAI. New research has found men fasting during the month of Ramadan recorded reduced bodyweight, body mass index, waist circumference to height ratio, and body adiposity index (BAI), with no apparent modification in the VAI. ${ }^{41}$ According to the study done by Amato MC et al ${ }^{42}$, anthropometric, metabolic and clinical parameters were studied in 92 Type 2 DM patients and the study concluded that the VAI as compared to other measures of assessment of adiposity assessments showed the finest correlation with cardio metabolic risk markers. ${ }^{42}$

In a study conducted by Kang YM et all3; around 2204 Korean subjects with the MHO-phenotype were engaged and classified based on metabolic health state and body mass index under Wildman criteria at initial and advanced examinations and the study concluded that MHO phenotypes exhibiting higher VAI values were indicative of relatively poor metabolic outcomes in the future.43

A study was done by Chen HY et al with 464 haemodialysis patients, it was concluded that VAI is the best suited method to measure visceral adiposity and correlates well with long-term CV outcomes and all-cause mortality in haemodialysis patients. Its predictive power of $\mathrm{CV}$ outcomes is better than WC and WHtR. ${ }^{4}$ VAI is an evidence-based tool to evaluate adipose tissue dysfunction and its associated cardio metabolic risk in various patient populations.

\section{Bioelectrical Impedance Analysis}

Bioelectrical Impedance Analysis (BIA) is an index used to measure body impedance by using electrodes that are connected to form a circuit for the electric current passage. The measure of impedance is used to estimate the total body water and fat-free mass. Varied tissues present diverse degrees of resistance. However, due to its low water content adipose tissue is a poor conductor of electric current. ${ }^{45-47}$ Since it is non-invasive, it requires a limited number of instruments and is cost-effective. BIA is an interesting index that presents no height or weight restrictions. Tetra and Eight Polar BIA also known as Segmental Bioelectrical Impedance Analysis Index recognizes the complexities in human shapes and further combines many impedance measures to derive an accurate analysis. ${ }^{47}$ BIA equations specific to fatness which were formulated by Segal et al. have been verified for handling obese and newly developed prediction equations derived for the obese are much more accurate to predict body fat. ${ }^{48,49}$

\section{Dual X-Ray Absorptiometry}

Dual X-ray Absorptiometry (DEXA) is a screening method that evaluates the bone mineral, fat tissue, and also fat-free soft tissue. It is beneficial in forecasting intra-abdominal fat in all obese women and men. DEXA also permits interpretation of the composition of body that can ultimately differentiate between android or gynoid obesity. Constraints of DEXA are its high cost, skilled technician's requirement and devoted facilities. ${ }^{50-51}$

\section{CONCLUSIONS}

There are several methods of assessment of body composition. The various tools and techniques available have their own drawbacks. New methods seem to be more committed for an efficient assessment of obesity, but these emerging tools should be validated by more research. When an attempt is being made to choose an ideal tool for analysing the body composition, clinicians should consider the resources available to them, cost effectiveness of the tool, and most importantly the goals of their ultimate management plan.

Financial or Other Competing Interests: None. 


\section{REFERENCES}

[1] Mhurchu CN, Rodgers A, Pan WH, et al. Body mass index and cardiovascular disease in the Asia-Pacific Region: an overview of 33 cohorts involving 310000 participants. Int J Epidemiol 2004;33(4):751-8.

[2] Keys A, Fidanza F, Karvonen MJ, et al. Indices of relative weight and obesity. J Chron Dis 1972;25(6):329-43.

[3] Health implications of obesity. National Institutes of Health Concensus development conference statement. Ann Intern Med 1985;103(6 Pt 2):1073-77.

[4] WHO. Physical status: the use and interpretation of anthropometry: report of a World Health Organization expert committee. Geneva, Switzerland: World Health Organization 1995.

[5] Wellens RI, Roche AF, Khamis HJ, et al. Relationships between the body mass index and body composition. Obes Res 1996;4(1):35-44.

[6] Flegal KM, Shepherd JA, Looker AC, et al. Comparisons of percentage body fat, body mass index, waist circumference, and waist-stature ratio in adults. Am J Clin Nutr 2009;89(2):500-8.

[7] Rothman KJ. BMI-related errors in the measurement of obesity. Int J Obes (Lond) 2008;32 Suppl 3:S56-9.

[8] Romero-Corral A, Somers VK, Sierra-Johnson J, et al. Accuracy of body mass index in diagnosing obesity in the adult general population. Int $\mathrm{J}$ Obes (Lond) 2008;32(6):959-66.

[9] Keys A, Aravanis C, Blackburn H, et al. Coronary heart disease: overweight and obesity as risk factors. Ann Intern Med 1972;77(1):15-27.

[10] Chapman JM, Massey FJ. The interrelationship of serum cholesterol, hypertension, body weight, and risk of coronary disease. results of the first ten years' follow-up in the Los Angeles Heart Study. J Chron Dis 1964;17(10):933-49.

[11] Kip KE, Marroquin OC, Kelley DE, et al. Clinical importance of obesity versus the metabolic syndrome in cardiovascular risk in women: a report from the Women's Ischemia Syndrome Evaluation (WISE) study. Circulation 2004;109(6):706-13.

[12] Pischon T, Boeing H, Hoffmann K, et al. General and abdominal adiposity and risk of death in Europe. N Engl J Med 2008;359(20):2105-20.

[13] Ross R, Neeland IJ, Yamashita S, et al. Waist circumference as a vital sign in clinical practice: a consensus statement from the IAS and ICCR working group on visceral obesity. Nat Rev Endocrinol 2020;16(3):177-89.

[14] Rexrode KM, Carey VJ, Hennekens CH, et al. Abdominal adiposity and coronary heart disease in women. JAMA 1998;280(21):1843-8.

[15] Despres JP. Excess visceral adipose tissue/ectopic fat the missing link in the obesity paradox? J Am Coll Cardiol 2011;57(19):1887-9.

[16] Hsieh SD, Yoshinaga H. Waist/height ratio as a simple and useful predictor of coronary heart disease risk factors in women. Intern Med 1995;34(12):1147-52.

[17] Ashwell M, Lejeune S, McPherson K. Ratio of waist circumference to height may be better indicator of need for weight management. BMJ 1996;312(7027):377.
[18] Ashwell M, Gunn P, Gibson S. Waist-to-height ratio is a better screening tool than waist circumference and BMI for adult cardiometabolic risk factors: systematic review and meta-analysis. Obes Rev 2012;13(3):275-86.

[19] Paajanen TA, Oksala NK, Kuukasjarvi P, et al. Short stature is associated with coronary heart disease: a systematic review of the literature and a meta-analysis. Eur Heart J 2010;31(4):1802-9.

[20] Zhang C, Rexrode KM, van Dam RM, et al. Abdominal obesity and the risk of all-cause, cardiovascular, and cancer mortality: sixteen years of follow-up in US women. Circulation 2008;117(3):1658-67.

[21] Song X, Jousilahti P, Stehouwer CDA, et al. Comparison of various surrogate obesity indicators as predictors of cardiovascular mortality in four European populations. Eur J Clin Nutr 2013;67(12):1298-302.

[22] Pischon T, Boeing H, Hoffmann K, et al. General and abdominal adiposity and risk of death in Europe. N Engl J Med 2008;359(20):2105-20.

[23] Seidell JC. Waist circumference and waist/hip ratio in relation to all-cause mortality, cancer and sleep apnea. Eur J Clin Nutr 2010;64(1):35-41.

[24] Misra A, Garg A, Abate N, et al. Relationship of anterior and posterior subcutaneous abdominal fat to insulin sensitivity in nondiabetic men. Obes Res 1997;5(2):93-9.

[25] Sinha R, Dufour S, Petersen KF, et al. Assessment of skeletal muscle triglyceride content by (1)H nuclear magnetic resonance spectroscopy in lean and obese adolescents: relationships to insulin sensitivity, total body fat, and central adiposity. Diabetes 2002;51(4):1022-7.

[26] Katzmarzyk PT, Craig CL. Independent effects of waist circumference and physical activity on all-cause mortality in Canadian women. Appl Physiol Nutr Metal 2006;31(3):271-6.

[27] Yusuf S, Hawken S, Ounpuu S, et al. Obesity and the risk of myocardial infarction in 27,000 participants from 52 countries: a case-control study. Lancet 2005;366(9497):1640-9.

[28] Wang Y, Rimm EB, Stampfer MJ, et al. Comparison of abdominal adiposity and overall obesity in predicting risk of type 2 diabetes among men. Am J Clin Nutr 2005;81(3):555-63.

[29] Yusuf S, Hawken S, Ounpuu S, et al. Effect of potentially modifiable risk factors associated with myocardial infarction in 52 countries (the INTERHEART study): casecontrol study. Lancet 2004;364(9438):937-52.

[30] Pratyush DD, Tiwari S, Singh S, et al. Waist circumference cutoff and its importance for diagnosis of metabolic syndrome in Asian Indians: a preliminary study. Indian J Endocr Metab 2012;16(1):112-5.

[31] Snehalatha C, Viswanathan V, Ramachandran A. Cutoff values for normal anthropometric variables in Asian Indian adults. Diabetes Care 2003;26(5):1380-4.

[32] Ghosh JR, Bandyopadhyay AR. Abdominal circumference as a screening measure for type 2 diabetes. Kathmandu Univ Med J 2012;10(40):12-5.

[33] Krakauer NY, Krakauer JC. A new body shape index predicts mortality hazard independently of body mass index. PLoS One 2012;7(7):e39504. 
[34] Krakauer NY, Krakauer JC. Dynamic association of mortality hazard with body shape. PLoS One 2014;9(2):e88793.

[35] Song X, Jousilahti P, Stehouwer CDA, et al. Cardiovascular and all-cause mortality in relation to various anthropometric measures of obesity in Europeans. Nutr Metab Cardiovasc Dis 2015;25(3):295-304.

[36] Dhana K, Kavousi M, Ikram MA, et al. Body shape index in comparison with other anthropometric measures in prediction of total and cause-specific mortality. J Epidemiol Community Health 2016;70(1):90-6.

[37] Song X, Jousilahti P, Stehouwer CDA, et al. Comparison of various surrogate obesity indicators as predictors of cardiovascular mortality in four European populations. Eur J Clin Nutr 2013;67(12):1298-302.

[38] Maessen MF, Eijsvogels TM, Verheggen RJHM, et al. Entering a new era of body indices: the feasibility of a body shape index and body roundness index to identify cardiovascular health status. PLoS One 2014;9(9):e107212.

[39] Chang Y, Guo X, Chen Y, et al. A body shape index and body roundness index: two new body indices to identify diabetes mellitus among rural populations in northeast China. BMC Public Health 2015;15:794.

[40] Mazzuca E, Battaglia S, Marrone 0, et al. Gender-specific anthropometric markers of adiposity, metabolic syndrome and visceral adiposity index (VAI) in patients with obstructive sleep apnea. Journal of Sleep Research 2014;23(1):13-21.

[41] Celik A, Saricicek E, Saricicek V, et al. Effect of Ramadan fasting on serum concentration of apelin-13 and new obesity indices in healthy adult men. Med Sci Monit 2014;20:337-42.

[42] Amato MC, Pizzolanti G, Torregrossa V, et al. Visceral adiposity index (VAI) is predictive of an altered adipokine profile in patients with type 2 diabetes. PLoS One 2014;9(3):e91969.

[43] Kang YM, Jung CH, Cho YK, et al. Visceral adiposity index predicts the conversion of metabolically healthy obesity to an unhealthy phenotype. PLoS One 2017;12(6):e0179635.

[44] Chen HY, Chiu YL, Chuang YF, et al. Visceral adiposity index and risks of cardiovascular events and mortality in prevalent hemodialysis patients. Cardiovasc Diabetol 2014;13:136.

[45] Strain GW, Wang J, Gagner M, et al. Bioimpedance for severe obesity: comparing research methods for total body water and resting energy expenditure. Obesity (Silver Spring) 2008;16(8):1953-6.

[46] Shafer KJ, Siders WA, Johnson LK, et al. Validity of segmental multiple-frequency bioelectrical impedance analysis to estimate body composition of adults across a range of body mass indexes. Nutrition 2009;25:25-32.

[47] Kushner RF. Bioelectrical impedance analysis: a review of principles and applications. J Am Coll Nutr April 1992;11(2):199-209.

[48] Stolarczyk LM, Heyward VH, Van Loan MD, et al. The fatness-specific bioelectrical impedance analysis equations of Segal et al: are they generalizable and practical? Am J Clin Nutr 1997;66(1):8-17.

[49] Horie LM, Barbosa-Silva MCG, Torrinhas RS, et al. New body fat prediction equations for severely obese patients. Clin Nutr 2008;27(3):350-6.

[50] Kamel EG, McNeill G, Van Wijk MC. Usefulness of anthropometry and DXA in predicting intra-abdominal fat in obese men and women. Obes Res 2000;8(1):36-42.

[51] Pateyjohns IR, Brinkworth GD, Buckley JD, et al. Comparison of three bioelectrical impedance methods with DXA in overweight and obese men. Obesity (Silver Spring) 2006;14(11):2064-70. 